\title{
Prognostic value of microvascular occlusion MRI quantification in assessment of reperfused myocardial infarction
}

\author{
Rana T. M. Khafagy ${ }^{1 *}$, Wesam El Mozy ${ }^{2,3}$, Emad Hamed', Yasser Ibrahim Abd ElKhalek and Wahid Tantawy ${ }^{1}$
}

\begin{abstract}
Background: Reperfusion therapy in patients with acute myocardial infarction (AMI) can salvage the myocardium; however, successful restoration of the coronary artery patency is not always associated with adequate perfusion at the level of microvasculature, known as the no-reflow or microvascular occlusion (MVO). The primary objective of our prospective study was to assess, by cardiac magnetic resonance (CMR), the prognostic value of MVO size, and its impact on left ventricular (LV) remodeling in cases of reperfused AMI.

Thirty-three patients with AMI underwent cardiac MRI at 1.5-T scanner within 7 days (baseline) and 3 months (follow-up) after reperfusion. Patients with MVO were included where early gadolinium enhancement (EGE), late gadolinium enhancement (LGE), and cine sequences were acquired. The impact of MVO size on LV ejection fraction (EF\%) and LV volumes was quantitively analyzed.

Results: There was a significant inverse correlation between the MVO size \% of the LV mass (LVM) and the EF\% values measured at follow-up with a $P$ value of 0.000 , while a significant positive correlation was encountered between the MVO\% of LVM and both indexed end-systolic volume (ESVI) and indexed end-diastolic volume (EDVI) values measured at follow-up with $P$ values of 0.438 and 0.389 , respectively. MVO size was found to be a significant factor affecting the patient's outcome $(P=0.000)$ where MVO size of $>10 \%$ of the total LVM can be a predictor of a worse outcome and reduced EF\% at follow-up.
\end{abstract}

Conclusion: The prognostic value of MVO could be statistically determined with a cut off value to predict a possible good outcome using CMR.

Keywords: Microvascular occlusion (MVO), CMR, Prognosis, LV remodeling

\section{Background}

Myocardial infarction (MI) is a leading cause of death and disability worldwide; consequently, its accurate diagnosis is important, since it directs the clinical management and affects the patient's prognosis [1].

Reperfusion therapy in patients with acute myocardial infarction (AMI) can salvage the myocardium and reduce mortality. However, successful restoration of the coronary artery patency does not always mean that

\footnotetext{
* Correspondence: Ranatarek@hotmail.com

${ }^{1}$ Radiodiagnosis Department_Faculty of Medicine, Ain Shams University, Cairo, Egypt

Full list of author information is available at the end of the article
}

perfusion of the microvasculature is adequately reached leading to what is known as the "no-reflow" or "microvascular occlusion" (MVO) [2].

While multiple different imaging modalities can diagnose the MVO, cardiac magnetic resonance (CMR) has many distinct advantages; it enables precise delineation of both infarct and MVO sizes along with accurate quantification of left ventricular (LV) function and volumes using a single imaging examination with no need for radiation exposure [3].

This study aims to evaluate, by CMR, the impact of MVO size on the regional LV wall characteristics and its

\section{Springer Open}

(c) The Author(s). 2020 Open Access This article is licensed under a Creative Commons Attribution 4.0 International License, which permits use, sharing, adaptation, distribution and reproduction in any medium or format, as long as you give appropriate credit to the original author(s) and the source, provide a link to the Creative Commons licence, and indicate if changes were made. The images or other third party material in this article are included in the article's Creative Commons licence, unless indicated otherwise in a credit line to the material. If material is not included in the article's Creative Commons licence and your intended use is not permitted by statutory regulation or exceeds the permitted use, you will need to obtain permission directly from the copyright holder. To view a copy of this licence, visit http://creativecommons.org/licenses/by/4.0/. 
local remodeling changes in cases of successfully reperfused acute myocardial infarction.

\section{Methods Subjects}

After obtaining approval from the Institutional Review Board, we conducted a single-center prospective study of AMI patients treated successfully by percutaneous intervention (PCI) reperfusion procedure within $12 \mathrm{~h}$ of acute symptoms' onset. Conventional coronary angiography (in the setting of PCI procedure) detected the culprit lesion at the left anterior descending (LAD) coronary artery in 16 patients (48.5\%), right coronary artery (RCA) in 10 patients (30.3\%), and left circumflex (LCx) coronary artery in 7 patients $(21.2 \%)$.

Thirty-three patients were included in our study $(28$ male, 5 females; mean age: $60.27 \pm 8.15$ years [range, 40-70 years]) (Table 1). All patients underwent a CMR examination within the first week of the reperfusion procedure and after 3 months in a period between June 2018 and May 2019. They or their guardians had signed the informed consent before proceeding for the examination.

We excluded patients who underwent reperfusion after $12 \mathrm{~h}$ from the onset of symptoms. Also, patients with previous cardiac events (infarction, thrombolysis), any significant valvular diseases, cardiomyopathies, GFR $<30 \mathrm{ml} / \mathrm{min} / 1.73 \mathrm{~m}^{2}$, or known to have contraindications for the MRI, e.g., an implanted magnetizable device, pacemakers, or claustrophobia, were excluded from our study.

\section{Methods}

The MRI study was performed on a 1.5-T scanner (Siemens machine Magnetom Aera, Siemens Medical Systems, Erlangen, Germany). All sequences were

Table 1 Patient characteristics

\begin{tabular}{ll}
\hline Patient age (years) & $60.27 \pm 8.15$ \\
\hline Patient gender & \\
$\quad$ Male & $n=28 / 33(84.8 \%)$ \\
Female & $n=5 / 33(15.2 \%)$ \\
Risk factors & \\
Current smoker only & $n=3 / 33(91 \%)$ \\
Hypertension only & $n=4 / 33(12.1 \%)$ \\
Diabetes mellitus only & $n=2 / 33(6 \%)$ \\
Mixed factors & $n=20 / 33(60.6 \%)$ \\
Infarct-related LAD artery & $16 / 33(48.5 \%)$ \\
Infarct-related RCA & $10 / 33(30.3 \%)$ \\
Infarct-related LCx artery & $7 / 33(21.2 \%)$. \\
\hline
\end{tabular}

ECG- and respiratory-gated with dedicated phased array cardiac coil using a single breath-hold multiecho technique. The examination time was $30-45$ min.

The examination protocol included retrospective ECG-gated cine steady-state free precession (SSFP) sequences during breath-hold in long-axis 2 and 4 chamber views (single slice at mid-LV, TR/TE $=72.75 /$ $1.2 \mathrm{~ms}$ ) and short-axis (SA) stack images covering both ventricles from the base of the heart to the apex for ventricular function assessment $(\mathrm{TR} / \mathrm{TE}=72.75 / 1.2$ $\mathrm{ms})$. SA-T2W fluid suppression stack images were acquired, using a prospective segmented inversionrecovery gradient-echo pulse sequence, for myocardial edema assessment $(\mathrm{TR} / \mathrm{TE}=800 / 52 \mathrm{~ms})$.

T1-weighted SA-early gadolinium enhancement (EGE) stack images, obtained 2-3 min after administration of a gadolinium-based contrast agent (Dotarem, Paris, France, $0.2 \mathrm{mmol} / \mathrm{kg}$ ), were acquired using a prospective segmented inversion-recovery gradient-echo pulse sequence $(\mathrm{TR} / \mathrm{TE}=547 / 3.14 \mathrm{~ms})$, inversion time was set to be $500 \mathrm{~ms}$. Also, T1-weighted SA-late gadolinium enhancement (LGE) stack images, obtained $10 \mathrm{~min}$ after injection of the contrast agent, were acquired using also a prospective segmented inversion-recovery gradientecho pulse sequence for myocardial fibrosis and $\mathrm{MVO}$ assessment $(\mathrm{TR} / \mathrm{TE}=654 / 1.06 \mathrm{~ms})$. An initial inversion time scout sequence was performed to determine the optimum inversion time.

Left ventricular function was analyzed by dedicated software (Philips intelliSpace portal workstation, version 8.0.) using a previously validated method [4]. Enddiastolic volume, end-systolic volume, and ejection fraction were all normalized to body surface area using the Mostellar formula [5, 6].

The left ventricular myocardial infarct and MVO volume quantification were performed by manually defining areas of enhancement on all the short-axis slices from the base to the apex using Segment CMR software, version 2.0, Mediviso.

\section{Statistical analysis}

For all volumetric measurements, the means and SD are quoted, with ranges from the $95 \%$ confidence intervals. Data were analyzed by SPSS statistical analysis program (SPSS version 23). It was summarized using descriptive statistics: number and percentage for qualitative values and mean and standard deviation, and median and interquartile range for quantitative variables. The paired-sample $t$ test was used to compare differences in global LV parameters between baseline and follow-up examinations and the independentsample $t$ test was used to compare mean values 
between subgroups. All statistical tests were two-tailed and a $P$ value of less than 0.05 was considered to indicate a significant difference.

\section{Results}

Forty patients met the inclusion criteria. Four patients were excluded due to claustrophobia, 2 patients refused to follow-up, and 1 patient died before completing his follow-up due to a non-cardiac cause. So 33 patients could complete the baseline and follow-up scans and were included in our statistical analysis. No cardiac deaths or major cardiac adverse events were noticed during the 3-months follow-up period.

Included patients $(n=33)$ were screened twice, within 1 week of the reperfusion procedure as a baseline scan and after 3 months as a follow-up scan, the infarct size $\%$ of the left ventricular mass (LVM) and MVO size \% of the LVM were calculated in the baseline scan. The median MVO size \% of LVM was 10 (1-43) and the mean scar size \% of LVM was $41.00 \pm 15.42(19-74)$. All patients showed complete resolution of the MVO at the follow-up scan.

The LV EF\%, LV end-diastolic volume index (EDVI), LV end-systolic volume index (ESVI), and LV stroke volume index (SVI) values were calculated in the baseline and follow-up scans and were compared to each other. Compared to the baseline scan, there was a significant increase in the follow-up scan values as regards the mean EDVI $(P=0.000)$, mean ESVI $(P$ $=0.005)$, and mean SVI $(P=0.007)$ using a paired $t$ test; while a significant decrease in the mean EF\% values was encountered at follow-up $(P=0.001)$ (Table 2, Figs. 1 and 2).

There was a significant positive correlation between the size of the MVO and the infarct measured at the baseline scan $(P$ value $=0.000)$ and a significant inverse correlation between the MVO size \% of the LVM and the $\mathrm{EF} \%$ values measured at the follow-up scans ( $P$ value of 0.000), while a significant positive correlation was encountered between the MVO\% of LVM and both ESVI and EDVI values measured in the follow-up scans with $P$ values of $0.011,0.025$, respectively (Table 3; Figs. 3,4 , and 5 ).

Based on the follow-up EF\% values, our patients were divided into 2 groups; a good-outcome group $(n=18$, 54.5), representing good prognosis, where the EF\% was stationary or improved in comparison to those measured in the baseline scan (Figs. 6 and 7), and a poor-outcome group ( $n=15,45.5 \%)$, representing bad prognosis, where the EF\% was reduced (Figs. 8 and 9). The MVO size \% of the LVM was found to be significantly affecting the outcome with $P=0.000$ using an independent $t$ test (Fig. 10).

Finally, a ROC curve for the predictors of a good outcome was statistically generated revealing that MVO size of $<10 \%$ of LVM is the cut off value for possible good prognosis with $94.44 \%$ sensitivity and $100 \%$ specificity (Fig. 11).

\section{Discussion}

MVO was first described in the 1970s [7]. It is usually encountered in up to $30 \%$ of the patients, as a reperfusion injury, following prolonged myocardial ischemia; the perfusion of the ischemic myocardium at the microvascular level is either unrestored or incompletely restored [2].

The pathophysiological mechanisms resulting in MVO development remain ill-defined; previous studies suggested multiple factors that can explain the mechanism of its formation [7, 8]. Swollen endothelial cells with intravascular luminal protrusions together with swollen myocytes occlude the capillaries. Also, the activation of neutrophils together with the platelets' deposition of fibrin contributes to microvascular damage. In addition to these factors, microvascular capillaries embolization with the atherosclerotic debris following the PCI procedure play an important role in microvascular capillaries' obstruction [2].

Table 2 Paired comparison of LVEF\% and LV volumes at 1st (baseline) and 2nd (follow-up) visits in all cases ( $n=33$ )

\begin{tabular}{|c|c|c|c|c|c|c|}
\hline & & 1st visit (baseline) & 2nd visit (follow-up) & Test value ${ }^{\circ}$ & $P$ value & Sig. \\
\hline \multirow[t]{2}{*}{$\mathrm{EF} \%$} & Mean \pm SD & $40.76 \pm 10.27$ & $38.06 \pm 12.50$ & 3.700 & 0.001 & HS \\
\hline & Range & $24-59$ & $15-59$ & & & \\
\hline \multirow[t]{2}{*}{ SVI } & Mean \pm SD & $33.44 \pm 9.94$ & $38.02 \pm 10.40$ & -2.861 & 0.007 & HS \\
\hline & Range & $20-70$ & $14-57$ & & & \\
\hline \multirow[t]{2}{*}{ EDVI } & Mean \pm SD & $85.67 \pm 24.00$ & $103.30 \pm 29.40$ & -5.451 & 0.000 & HS \\
\hline & Range & 49-173 & $33-179$ & & & \\
\hline \multirow[t]{2}{*}{ ESVI } & Mean \pm SD & $52.50 \pm 21.66$ & $62.38 \pm 29.00$ & -3.046 & 0.005 & HS \\
\hline & Range & $23-120$ & $15.5-135$ & & & \\
\hline
\end{tabular}

$P$ value $>0.05$, non-significant; $P$ value $<0.05$, significant; $P$ value $<0.01$, highly significant

'Paired $t$ test 


\section{口Baseline DFollow-up}

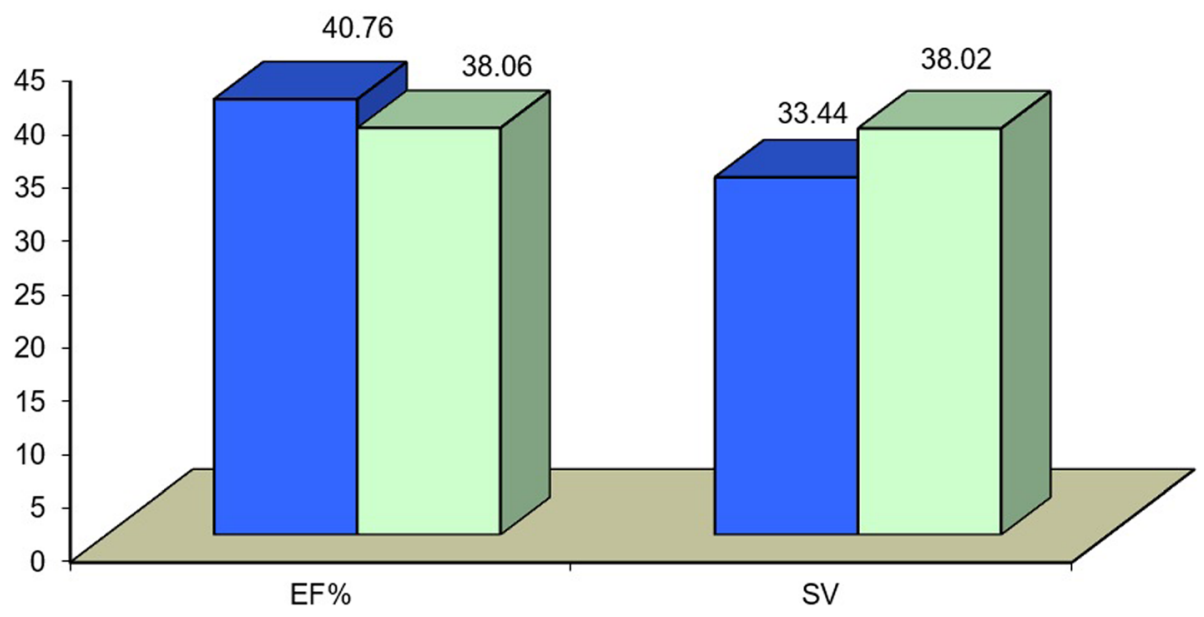

Fig. 1 A chart showing LV EF\% and SVI measured at 1st (baseline) and 2nd (follow-up) visits

MVO can be demonstrated via multiple imaging modalities; CMR is a valid tool that can accurately diagnose and quantify MVO. The major benefits of using CMR include the absence of radiation exposure, safety, being a non-invasive method, and a reproducible examination [9].

Studies showed that the extent of MVO, seen on CMR, shows a close correlation with the anatomical extent of MVO in both animals and humans. MVO size increases over the first 2 days following the $\mathrm{PCI}$ procedure and starts to reduce in size 2 to 7 days afterward [10]; most of the patients demonstrate complete resolution of MVO when screened 4 to 6 weeks post-PCI [3]. Consequently, in this study, we screened the patients within 1 week and 3 months of the post-reperfusion procedure. More important, MVO assessed on LGE imaging seems to have superior prognostic value to that assessed at early imaging besides it has a good spatial resolution [11]. Thus, in the present study, MVO was quantitively analyzed at the LGE images. It represents a central "dark

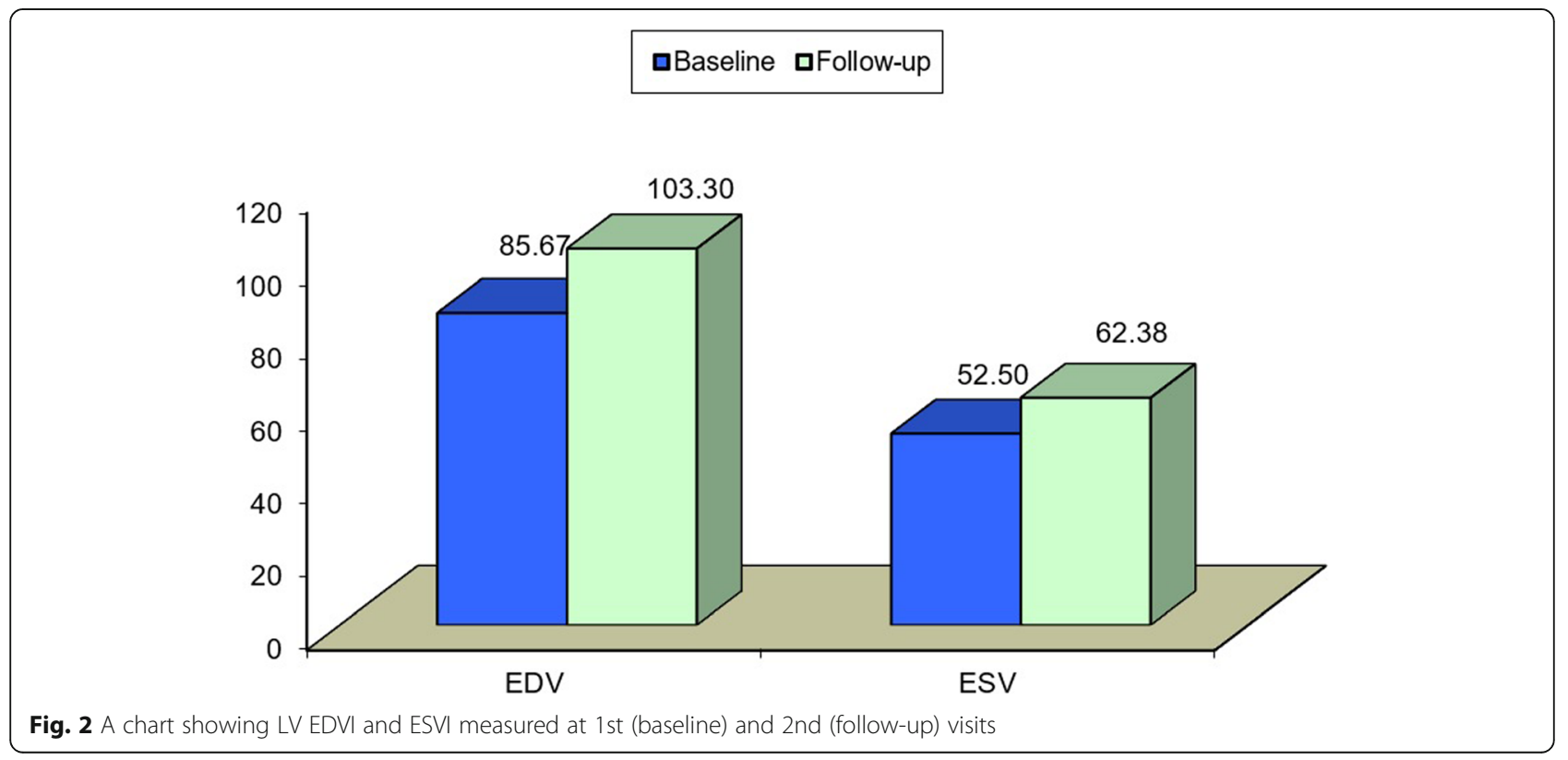


Table 3 Correlation between MVO size \% and baseline scar size together with LV volumes measured at 2nd visit (follow-up) and its significance

\begin{tabular}{lll}
\hline & \multicolumn{2}{l}{ All cases $n=33$} \\
\cline { 2 - 3 } & MVO \% & \\
\cline { 2 - 3 } & $r$ & 0.000 \\
\hline Scar size (at baseline scan) & +0.652 & 0.000 \\
EF\% (follow-up) & -0.582 & 0.636 \\
SVI (follow-up) & -0.086 & 0.025 \\
EDVI (follow-up) & +0.389 & 0.011 \\
ESVI (follow-up) & +0.438 &
\end{tabular}

$P$ value $>0.05$, non-significant; $P$ value $<0.05$, significant; $P$ value $<0.01$, highly significant Spearman correlation coefficient

$r<0.3$, no or very weak correlation; $r>0.3-<0.5$, weak correlation; $r>0.5-<$

0.7 , moderate correlation; $r>0.7$, strong correlation. " + " sign indicates positive correlation, "-" sign indicates negative correlation

zone" within the hyper-enhanced region on gadoliniumenhanced MRI sequences.

Though conflicting data still exist concerning the MVO presence and regional function recovery [11], yet multiple studies have shown that MVO has a significant adverse prognostic impact on the LV remodeling $[8,12]$ and that patients with MVO demonstrate greater left ventricular end-diastolic and end-systolic volumes at follow-up visits [10, 12-14]. We managed to confirm these findings in our study where we detected a significant increase in the follow-up scan values as regards the mean EDVI, the mean ESVI, and the mean SVI. Besides, we detected a significant inverse correlation between the MVO size \% of the LVM and the followup EF\% values together with a significant positive correlation between the MVO size \% of LVM and both ESVI and EDVI values measured in the followup scans.

The regional systolic function can be an independent predictor for patient outcome after MI [15] So, we divided the patients of this study into a good-outcome group $(n=18,54.5 \%)$, representing possible good prognosis, where the follow-up EF\% was stationary or improved in comparison to those measured in the baseline scan, and a poor-outcome group $(n=15,45.5 \%)$, where the follow-up EF\% was reduced; the MVO size in this study was found to be a significant factor affecting the type of outcome. More important, an MVO size of $<10 \%$ of LVM was found to be statistically the cut off value for a possible good outcome with high sensitivity $(94.44 \%)$ and specificity (100\%). Thus, the

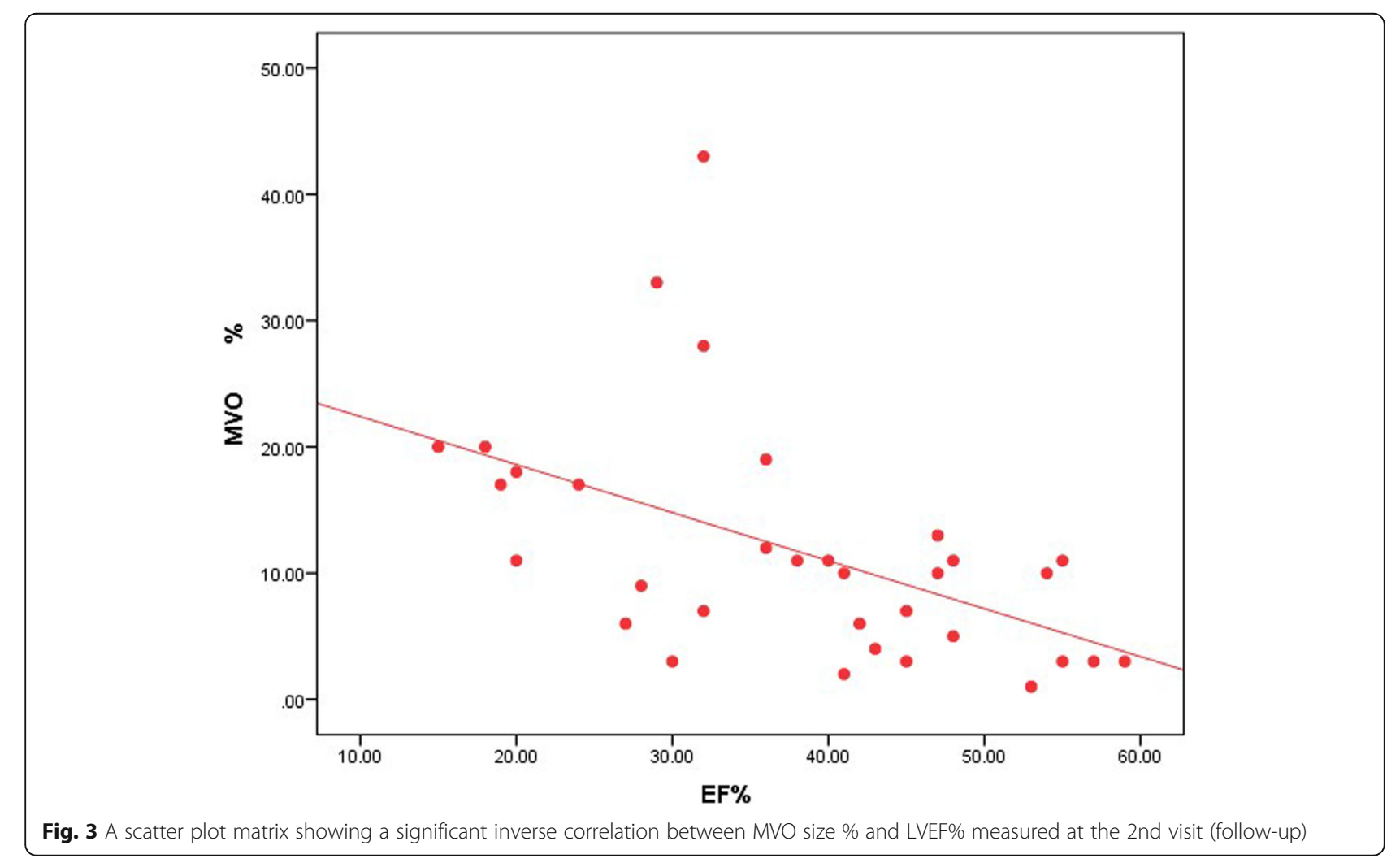




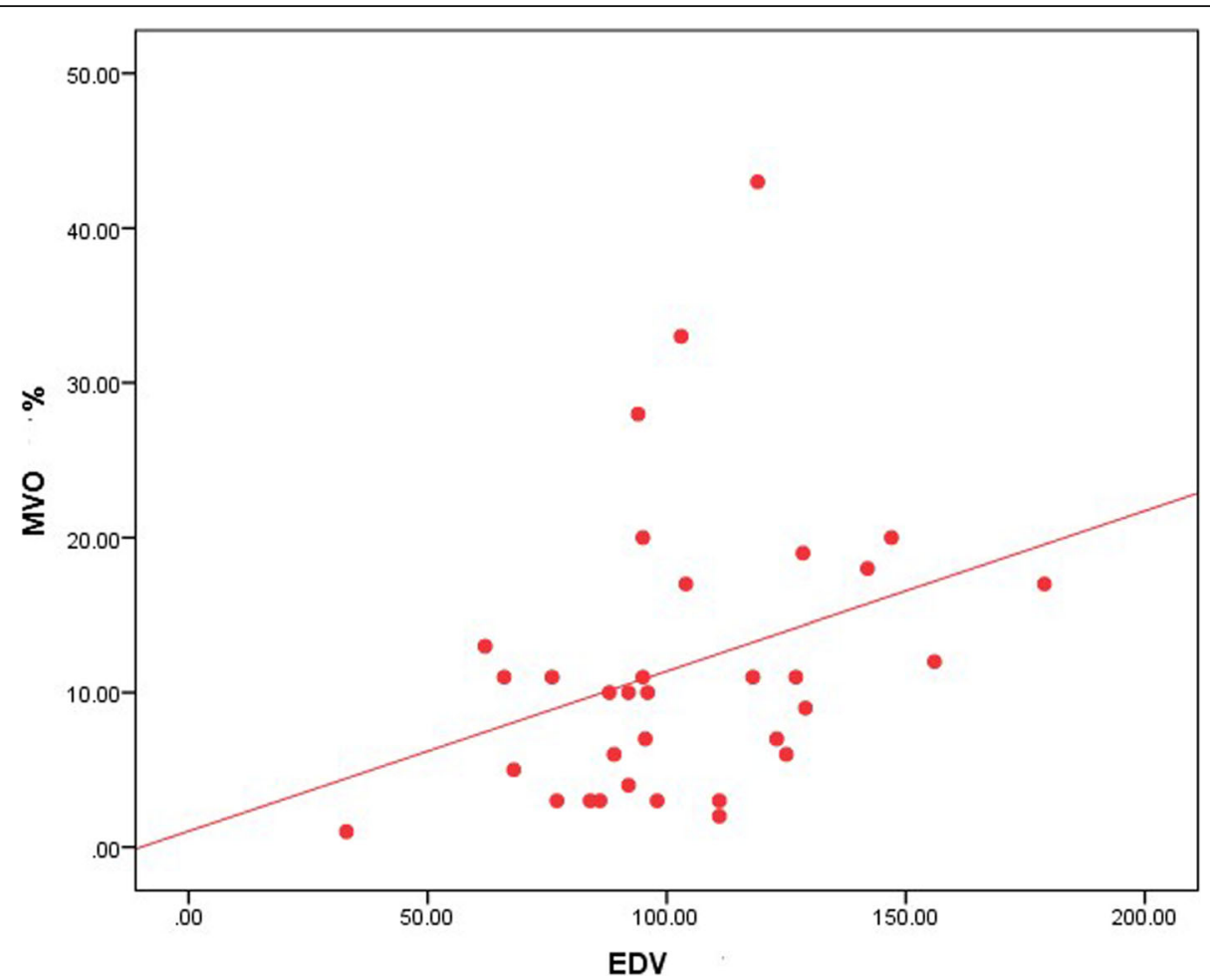

Fig. 4 A scatter plot matrix showing a significant positive correlation between MVO size \% and LV EDVI measured at the 2nd visit (follow-up)

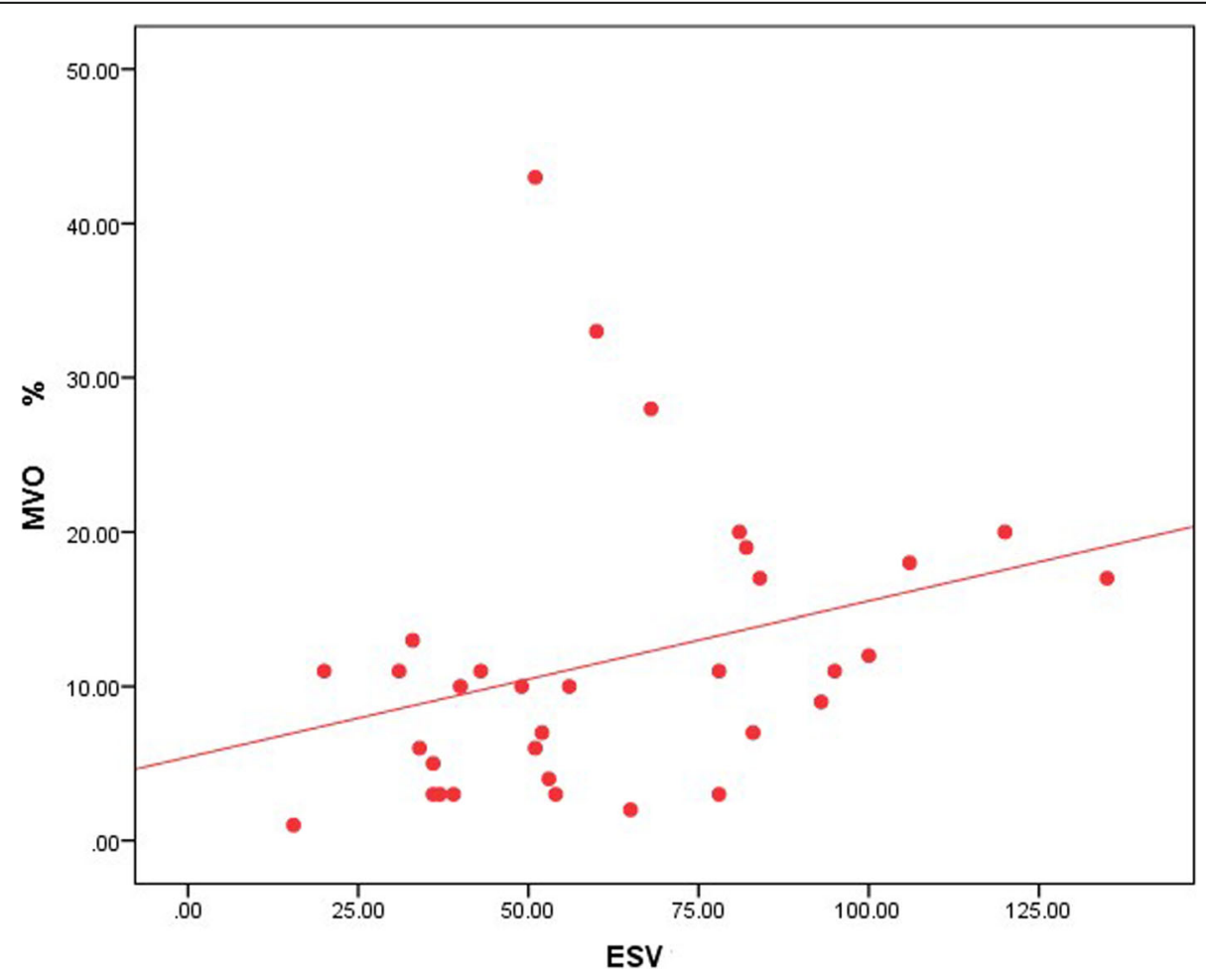

Fig. 5 A scatter plot matrix showing a significant a positive correlation between MVO size \% and LV ESVI measured at the 2nd visit (follow-up) 


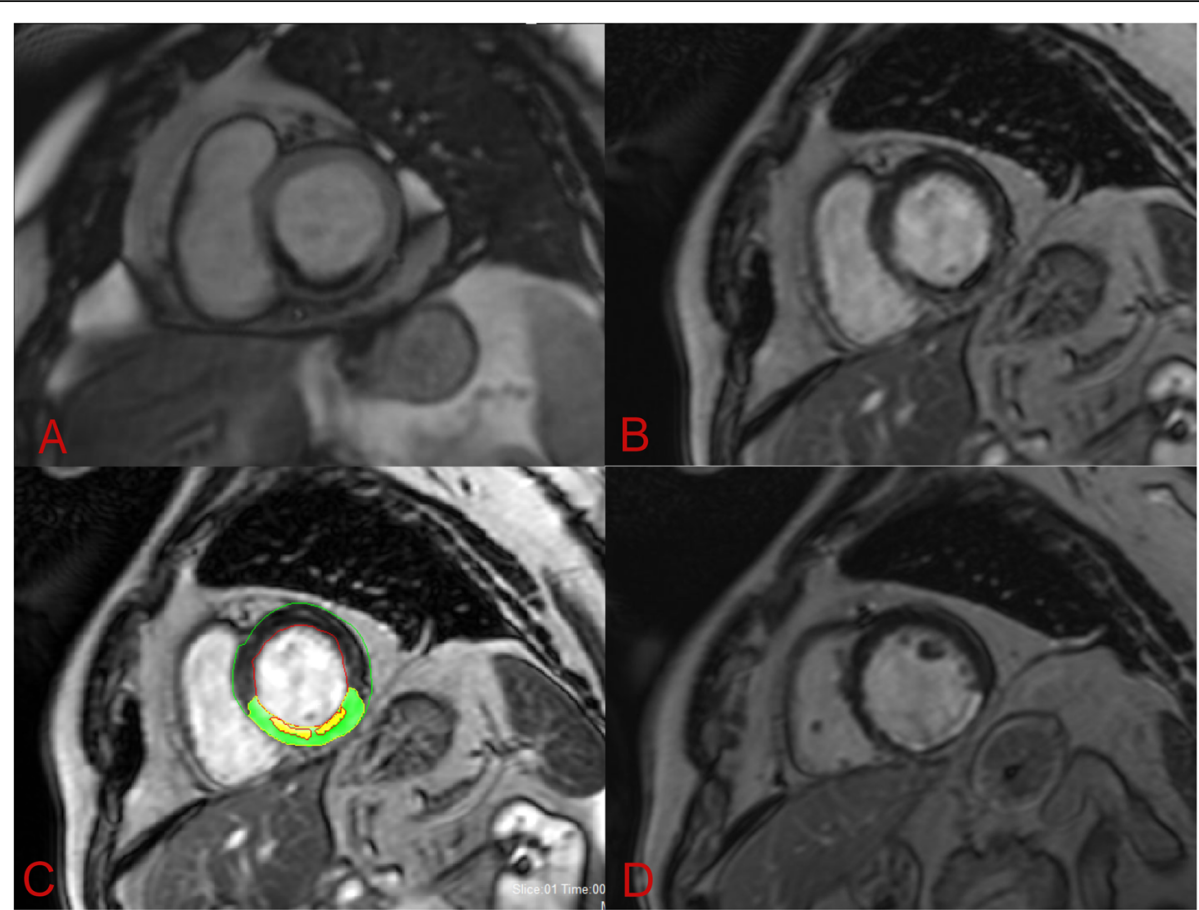

Fig. $\mathbf{6}$ a Cardiac MR performed for a patient 4 days post-primary percutaneous coronary intervention. Short-axis EGE sequence at mid-ventricular level shows a sub-endocardial inferior and infero-septal left ventricular wall low signal corresponding to microvascular obstruction. b Corresponding shortaxis LGE sequence showing transmural inferior and infero-septal left ventricular wall hyperenhancement/infarct with central low signal microvascular obstruction. Culprit lesion was detected at RCA. c Corresponding short-axis T1W LGE sequence with post-processing of the LV scar (green) and MVO (yellow) revealing an MVO size $=9 \%$ and infarct size $=27 \%$ of the total left ventricular mass (LVM). Follow-up short-axis LGE sequence, after 3 months, showing transmural inferior and sub-endocardial infero-septal left ventricular wall fibrosis scar with complete resolution of the MVO

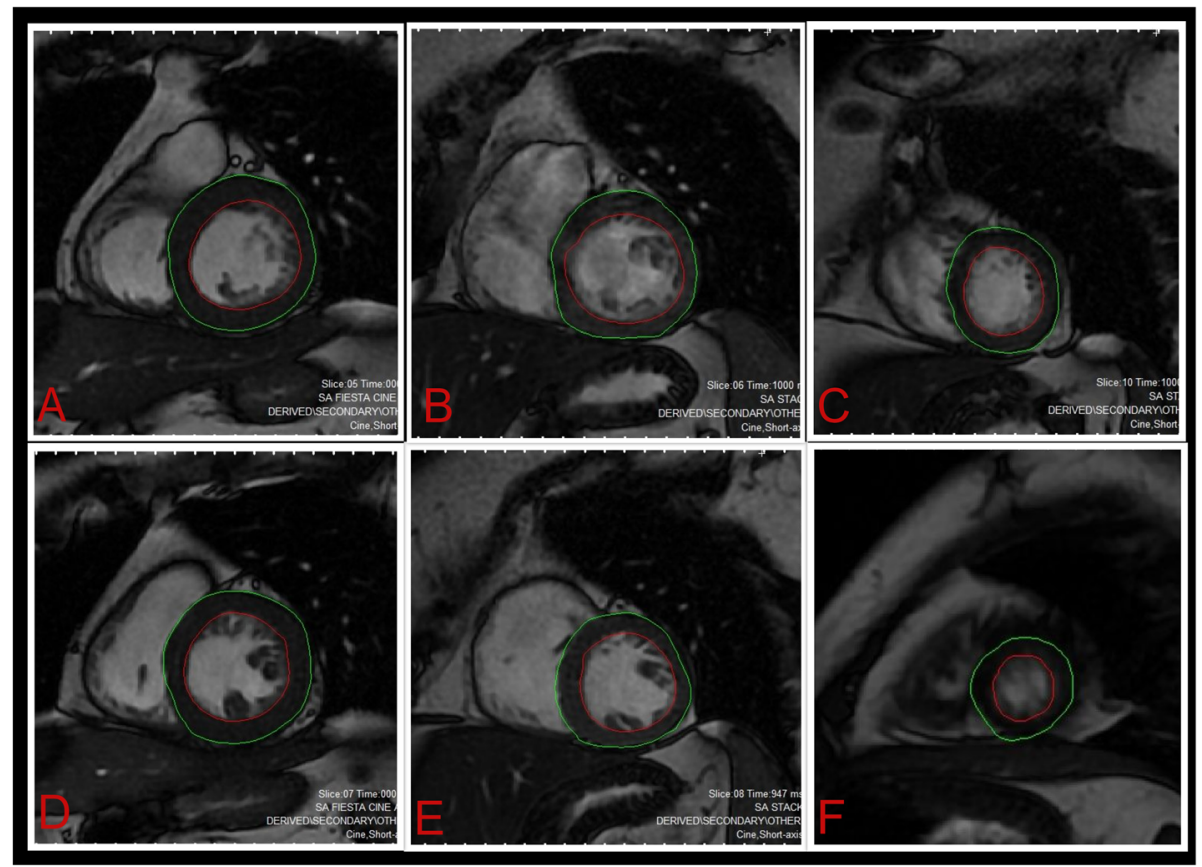

Fig. 7 Represent LV functional post-processing for the SSFP cine images taken for the previous patient; the endocardial (red) and epicardial borders (green) are manually delineated to obtain LV volumes, mass, and EF. a-c Basal, mid, and apical cuts at the baseline scan, calculated EF\% was $56 \%$. d-f Basal, mid, and apical cuts for the same patient acquired at the follow-up scan, calculated EF\% was $54 \%$ 


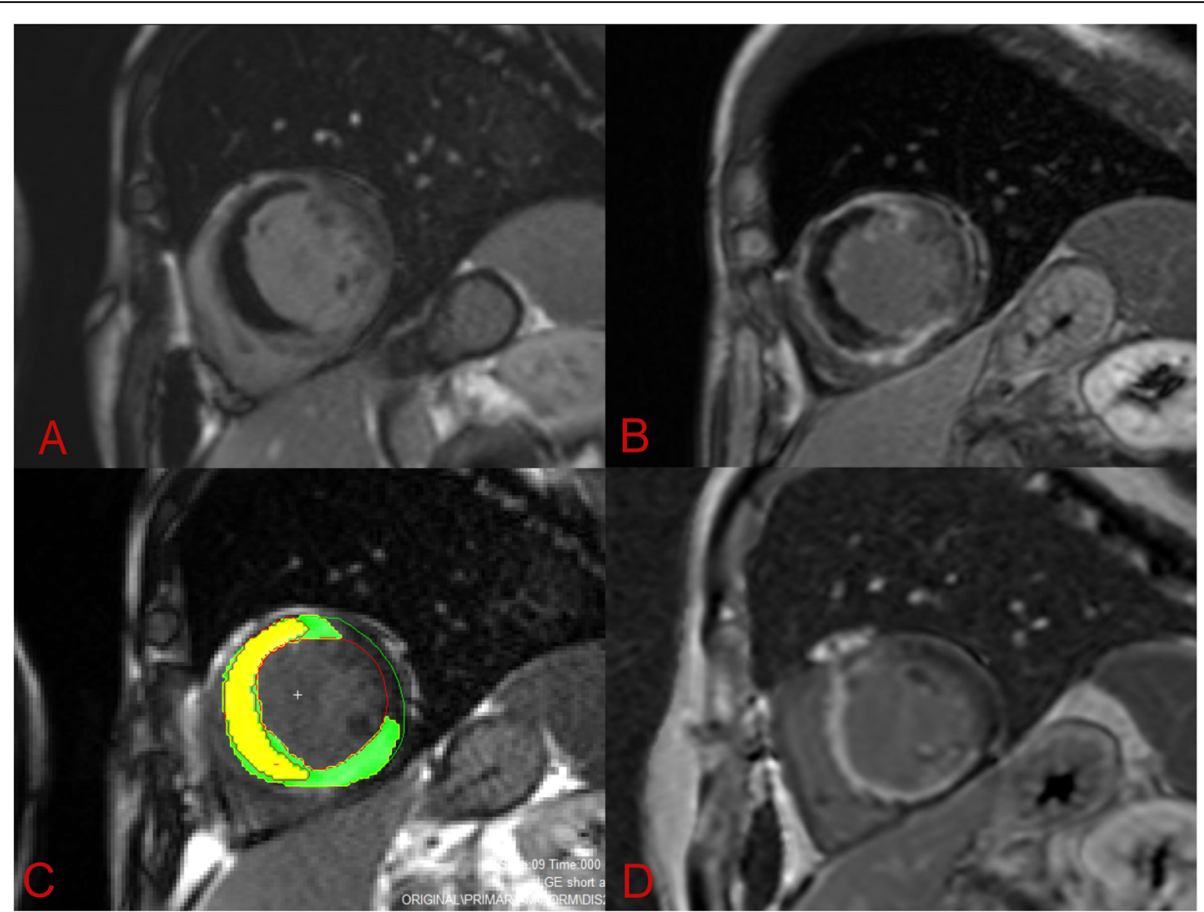

Fig. 8 a Cardiac MR performed for a patient 5 days post-primary percutaneous coronary intervention. Short-axis EGE sequence showing anterior and entire septal low signal corresponding to a large region of microvascular obstruction. $\mathbf{b}$ Corresponding short-axis LGE sequence showing fullthickness hyperenhancement/infarct involving anterior, entire septal, and inferior left ventricular wall. The septal low signal within the infarct corresponds to a large region of microvascular obstruction. Culprit lesion was detected at LAD artery. c: Corresponding Short-axis LGE sequence with post-processing of the LV scar (green) and MVO (yellow) revealing an MVO size $=43 \%$ and scar size $=93 \%$ of the total left ventricular mass (LVM). d Follow-up short-axis LGE sequence, after 3 months, transmural anterior, transmural septal, and sub-endocardial inferior left ventricular wall fibrosis scar with complete resolution of the MVO

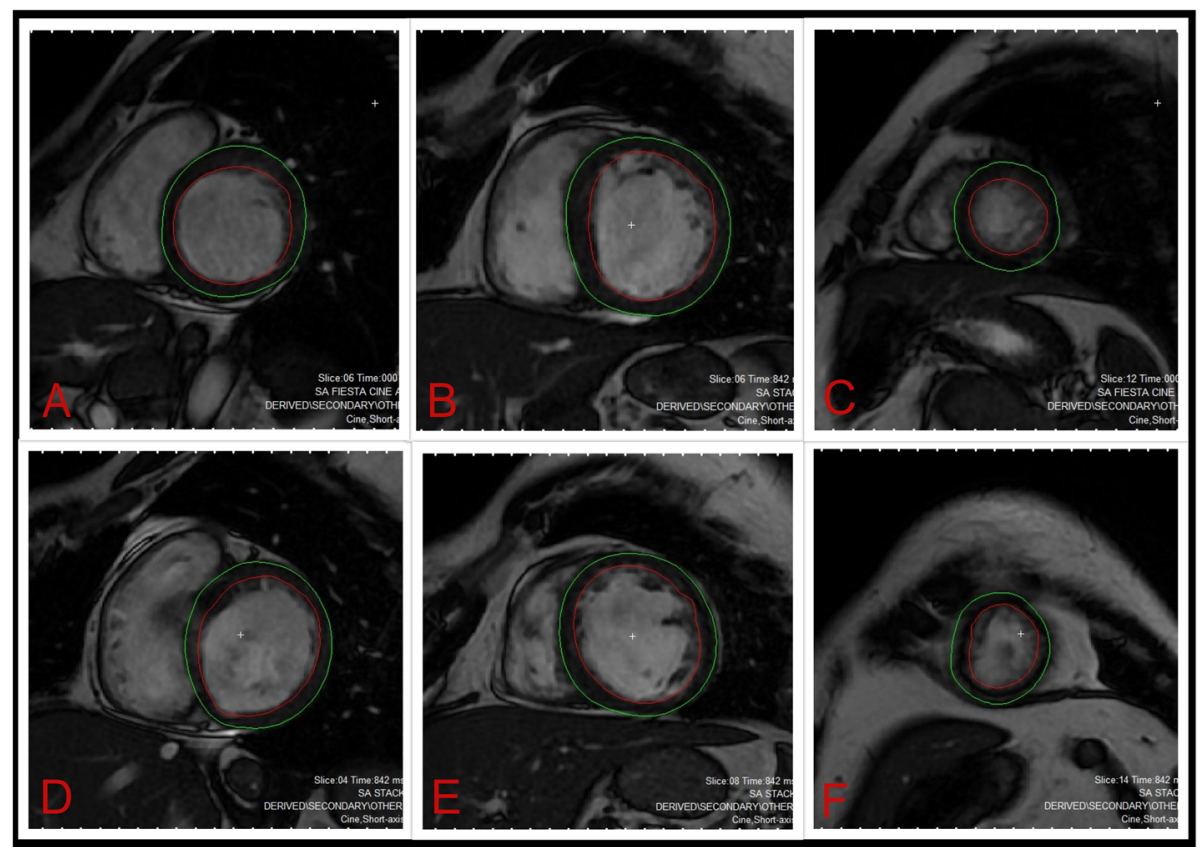

Fig. 9 Represent LV functional post-processing for the SSFP cine images taken for the previous patient; the endocardial (red) and epicardial borders (green) are manually delineated to obtain LV volumes, mass, and EF. a-c Basal, mid, and apical cuts at the baseline scan, calculated EF\% was $35 \%$. d-f Basal, mid, and apical cuts for the same patient acquired at the follow-up scan, calculated EF\% was $28 \%$ 


\section{Outcome}

\section{Poor aGood}
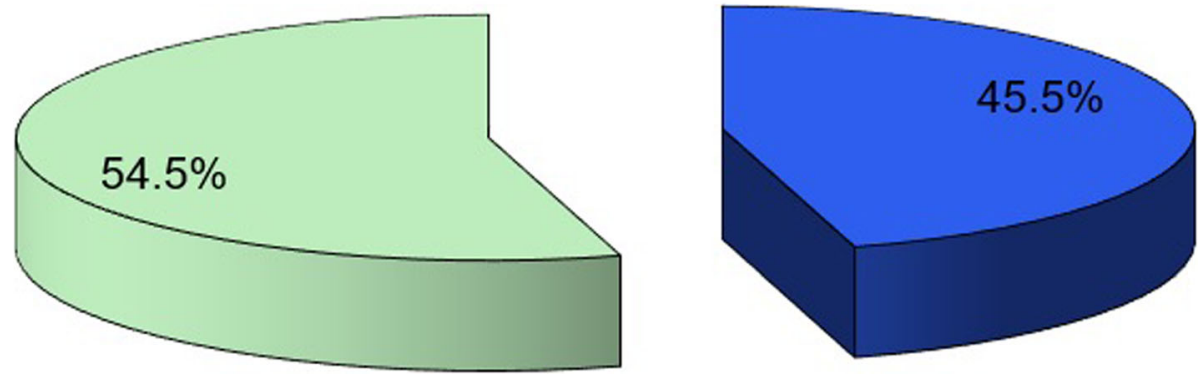

Fig. 10 A pie chart showing the percentage of good-and poor-outcome patients

prognostic value for the MVO size could be suggested in our study.

Several limitations of this study should be mentioned. The studied population is relatively small in size with no control group for comparison. We only used the EDVI, EDSVI, SVI, and EF\% values as determinants for LV remodeling and the patients' prognosis. Also, we did not perform $\mathrm{T} 2$ or $\mathrm{T} 2 * \mathrm{MR}$ sequences to detect intramyocardial hemorrhage, so its effect could not be assessed.

\section{Conclusion}

MVO size of $<10 \%$ of the LVM can be considered the cut off value for a possible good outcome. Thus, the prognostic value of MVO's size can be statistically determined using CMR. More studies are required to study local LV remodeling after AMI with a larger sample size and with broader spectrum; for example, comparing patient groups undergoing different reperfusion therapies, or exploring its evolution in multiple time points.

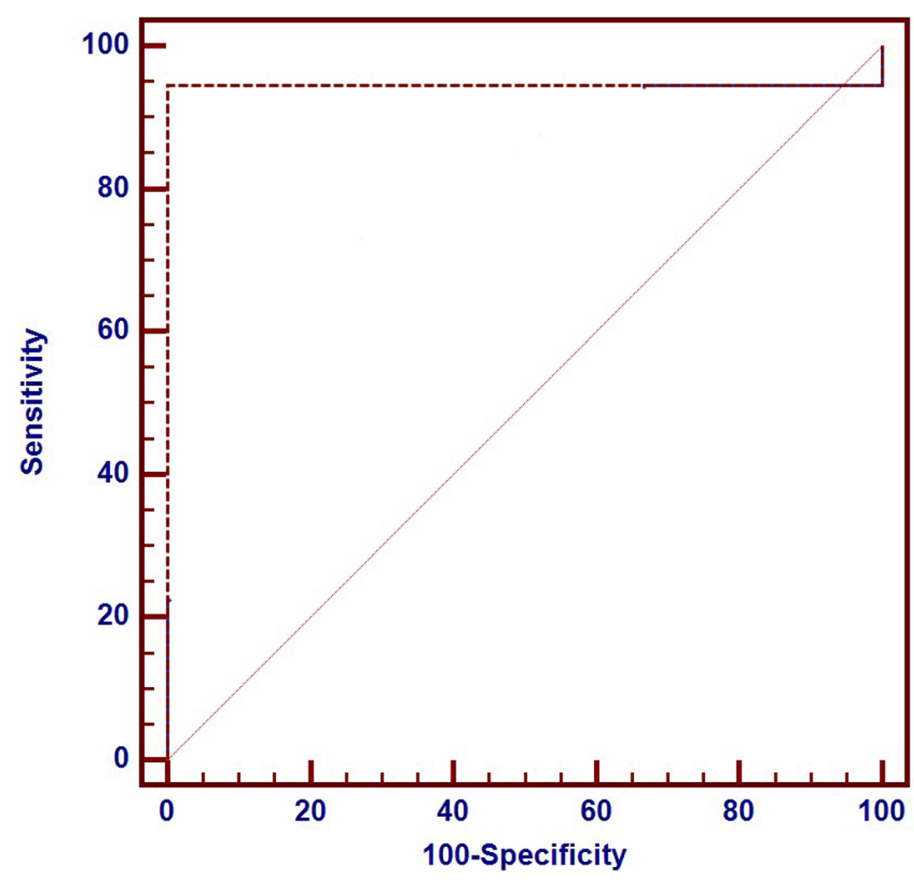

MVO ext $\%$

Fig. 11 ROC curve for the predictors of a good outcome 


\section{Abbreviations}

AMl: Acute myocardial infarction; MVO: Microvascular occlusion; LV: Left ventricular; EF\%: Ejection fraction; LVM: LV mass; EDVI: Indexed end-diastolic volume; ESVI: Indexed end-systolic volume; SVI: Indexed stroke volume; CMR: Cardiac MRI; SSFP: Steady-state free precession; SA: Short-axis; LGE: Late gadolinium enhancement; EGE: Early gadolinium enhancement; LAD: Left anterior descending coronary artery; RCA: Right coronary artery; LCx: Left circumflex coronary artery; $\mathrm{PCl}$ : Percutaneous intervention

\section{Acknowledgements}

The authors thank all the study participants for their patience and support.

\section{Authors' contributions}

RTMK: Reviewing literature, data collection and analysis, perform statistical analysis, write and revise the manuscript, and prepare figure and tables. WEM: Suggest and develop the research idea, share in data collection, reviewing literature, data analysis, share in statistical analysis, data analysis, and manuscript editing. EH: Reviewing literature, data analysis, and manuscript editing. YIA: Reviewing literature. WT: Reviewing literature, data analysis, and manuscript editing. All authors read and approved the final manuscript.

\section{Funding}

This study had no funding from any resource.

\section{Availability of data and materials}

The datasets used and/or analyzed during the current study are available from the corresponding author on reasonable request.

\section{Ethics approval and consent to participate}

Institutional (Ain Shams University) ethical clearance was taken before conducting this prospective study; June 2018-reference number 000017585.

The procedure was explained in full and the written informed consent was signed by the patient or his/her guardian.

\section{Consent for publication}

All patients included in this research gave written informed consent to publish the data contained within this study. If the patient was deceased or unconscious when consent for publication was requested, written informed consent for the publication of this data was given by their parent or legal guardian.

\section{Competing interests}

The authors declare they have no competing interests.

\section{Author details}

${ }^{1}$ Radiodiagnosis Department-Faculty of Medicine, Ain Shams University, Cairo, Egypt. ${ }^{2}$ Radiodiagnosis Department, Aswan Heart Center, Aswan, Egypt. ${ }^{3}$ Radiodiagnosis Department, Faculty of Medicine, Cairo University, Cairo, Egypt.

Received: 23 February 2020 Accepted: 12 May 2020

Published online: 29 May 2020

\section{References}

1. Jaarsma C, Schalla S, Cheriex E et al (2013) Incremental value of cardiovascular magnetic resonance over echocardiography in the detection of acute and chronic myocardial infarction. J Cardiovasc Magn Reson 15(5): $1-10$

2. Sebastiaan C, Yazdani SK et al (2010) Microvascular obstruction underlying pathophysiology and clinical diagnosis. J Am Coll Cardiol 55(16):1649

3. Abbas A, Matthews GH, Brown IW et al (2015) Cardiac MR assessment of microvascular obstruction. Br J Radiol 88(20):140-470

4. Tanner MA, Galanello R, Dessi C, Westwood MA, Smith GC, Nair SV Anderson LJ, Walker JW, Pennell DJ (2006) Myocardial iron loading in patients with thalassemia major on deferoxamine chelation. J Cardiovasc Magn Reson 8:543-547

5. Mosteller RD (1987) Simplified calculation of body surface area. N Engl J Med 317:1098
6. Maceira AM, Prasad SK, Khan M, Pennell DJ (2006) Normalized left ventricular systolic and diastolic function by steady-state free precession cardiovascular magnetic resonance. J Cardiovasc Magn Reson 8:417-426

7. Wu KC. (2011) CMR of microvascular obstruction and hemorrhage in myocardial infarction. J Cardiovasc Magn Reson 2012; 14: 68.

8. Schwartz BG, Kloer RA Coronary no reflow. J Mol Cell Cardiol 52:873-882

9. Greenwood J, Maredia N, Younger J et al (2012) Cardiovascular magnetic resonance and single-photon emission computed tomography for diagnosis of coronary heart disease (CE-MARC) a prospective trial. Lancet 379(9814):453-460

10. Mather AN, Fairbairn TA, Artis NJ, Greenwood JP, Plein S (2011) Timing of cardiovascular MR imaging after acute myocardial infarction: effect of estimates of infarct characteristics and prediction of late ventricular remodeling. Radiology 261:116-126

11. Lin Z, Mandry D, Chen B, Huttin O, Hossu G, Wang H et al (2018) Impact of microvascular obstruction on left ventricular local remodeling after reperfused myocardial infarction. J Magn Reson Imaging 47:499-510

12. Kidambi A, Mather AN, Motwani M, Swoboda P, Uddin A, Greenwood JP et al (2013) The effect of microvascular obstruction and intramyocardial hemorrhage on contractile recovery in reperfused myocardial infarction: insights from cardiovascular magnetic resonance. J Cardiovasc Magn Reson 15:58

13. Nijveldt R, Hofman MB, Hirsch A, Beek AM, Umans VA, Algra PR et al (2009) Assessment of microvascular obstruction and prediction of short-term remodelling after acute myocardial infarction: cardiac MR imaging study. Radiology 250:363-370

14. Weir RA, Murphy CA, Petrie CJ, Martin TN, Balmain S, Clements S et al (2010) Microvascular obstruction remains a portent of adverse remodeling in optimally treated patients with left ventricular systolic dysfunction after acute myocardial infarction. Circ Cardiovasc Imaging 3:360-367

15. Moller JE, Hillis GS, Oh JK, Reeder GS, Gersh BJ, Pellikka PA (2006) Wall motion score index and ejection fraction for risk stratification after acute myocardial infarction. Am Heart J 151:419-425

\section{Publisher's Note}

Springer Nature remains neutral with regard to jurisdictional claims in published maps and institutional affiliations.

\section{Submit your manuscript to a SpringerOpen ${ }^{\circ}$ journal and benefit from:}

- Convenient online submission

- Rigorous peer review

- Open access: articles freely available online

- High visibility within the field

- Retaining the copyright to your article

Submit your next manuscript at $\boldsymbol{\nabla}$ springeropen.com 